\title{
Robust Economic Model Predictive Control for Energy Dispatch in Smart Micro-grids Considering Demand Uncertainty
}

\author{
M. Nassourou ${ }^{1,2}$, J. Blesa ${ }^{1,2}$, V. Puig ${ }^{1,2}$ \\ ${ }^{1}$ Automatic Control Department, Technical University of Catalonia (UPC), \\ Carrer Pau Gargallo, 5, 08028 Barcelona (Spain). \\ ${ }^{2}$ Institut de Robòtica i Informàtica Industrial (CSIC-UPC), \\ Carrer Llorens Artigas, 4-6, 08028 Barcelona (Spain) \\ e-mail: vicenc.puig@upc.edu
}

\begin{abstract}
This paper proposes a robust economic model predictive control method based on a bounded description of the demand uncertainty for optimal energy dispatch in a smart micro-grid. The proposed method considers the effect of uncertainty by tightening the system constraints along the prediction horizon. The bounds of the tightened constraints are computed offline. A periodic terminal state constraint is included in order to guarantee stability. The proposed method is simpler and faster than closed-loop min-max based approaches. The proposed approach is assessed with the help of a smart-grid consisting of some photovoltaic (PV) panels, a wind generator, a hydroelectric generator, a diesel generator, and some storage devices interconnected via two DC-buses, from which load demands can draw required energy. Load demands are uncertain, but considered as bounded.
\end{abstract}

Keywords-Smart Grid, Economic MPC, Robustness, Zonotope, Uncertainty

\section{INTRODUCTION}

Automatic control of complex Multiple Input Multiple Output (MIMO) electrical systems such as smart grids requires advanced predictive control strategies. Model Predictive Control (MPC) is nowadays one of the most advanced and frequently used techniques for achieving optimal control of complex MIMO systems. Moreover, the complexity of smart grids increases with the presence of uncertainties. The problem of model uncertainty and noise can be solved through enforcement of computational constraints as reported in [1],[2],[4]. However, this approach raises usually the issue of computational tractability.

Optimization techniques such as Minimax MPC approaches as reported in [8] and [9] can better tackle uncertainties in complex systems. However, one of the main drawbacks of Minimax is that, they principally consider worst case scenarios.

Moreover, in [3][4] adjustable robust solutions have been proposed, which assume that adjustable control inputs can be made to depend affinely on the uncertainty parameters of the problem. This approach is more flexible, and is most of the time expected to result in a computationally tractable problem.
In this work, we propose a robust economic model predictive control method that considers the effect of demand uncertainty by tightening the system constraints along the prediction horizon. The bounds of the tightened constraints are computed offline. Additionally, a periodic terminal state constraint is included in order to guarantee stability. The proposed method is simpler and faster than closed-loop minmax based approaches, since all the computations do not need to be carried out online. In order to verify the applicability of the developed method, we consider a hybrid system comprising some photovoltaic (PV) panels, a wind generator, a hydroelectric generator, a diesel generator, and some storage devices interconnected via two DC-buses, from which load demands can be satisfied. Load demands are uncertain, but considered as bounded.

The structure of the paper is the following: Section II presents the problem formulation and the economic MPC strategy. Section III describes how to robustify the proposed MPC strategy against demand uncertainty. Section IV shows how to update the variable bounds of the MPC optimization problem to consider demand uncertainty. Section V illustrates the proposed approach with a smart grid case study. Section VI draws the main conclusions and presents future research paths.

\section{Problem Statement}

\section{A. Control-oriented modelling}

Smart grids can be considered as instances of generalized flowbased networks. Basically every flow-based network consists of some components [5],[6],[7],[8],[10],[12], e.g.: flow sources, links, nodes, storage, flow handling, and sink elements.

Let us consider a smart micro-grid consisting of $n_{x}$ storage elements, $n_{u}$ energy flow handling and source elements, $n_{d}$ sinks (demands) and $n_{q}$ intersection nodes. Then, for control purposes the smart grid can be represented by the following discrete-time descriptor linear model 


$$
\begin{aligned}
& \mathbf{x}(k+1)=\mathbf{A} \mathbf{x}(k)+\mathbf{B} \mathbf{u}(k)+\mathbf{B}_{\mathrm{d}} \mathbf{d}(k) \\
& \mathbf{E}_{\mathbf{u}} \mathbf{u}(k)+\mathbf{E}_{\mathrm{d}} \mathbf{d}(k)=0
\end{aligned}
$$

where:

$\mathbf{x} \in \mathfrak{R}^{\mathrm{n}_{\mathrm{x}}}$ is the state vector, $\mathbf{u} \in \mathfrak{R}^{\mathrm{n}_{\mathrm{u}}}$ stands for the vector of control inputs, $\mathbf{d} \in \mathfrak{R}^{\mathrm{n}_{\mathrm{d}}}$ denotes the disturbances (i.e. demands) vector. $\mathbf{A} \in \mathfrak{R}^{\mathrm{n}_{\times} \times \mathrm{n}_{\mathrm{x}}}, \mathbf{B} \in \mathfrak{R}^{\mathrm{n}_{\mathrm{x}} \times \mathrm{n}_{u}}, \quad \mathbf{B}_{d} \in \mathfrak{R}^{\mathrm{n}_{\mathrm{x}} \times \mathrm{n}_{d}}$ are system matrices. $\mathbf{E}_{u} \in \mathfrak{R}^{\mathrm{n}_{q} \times \mathrm{n}_{u}}$ and $\mathbf{E}_{d} \in \mathfrak{R}^{\mathrm{n}_{q} \times \mathrm{n}_{d}}$ are matrices of suitable dimensions relating energy suppliers and load demands on the $n_{q}$ DC bus(ses).

Assumption 1. The states $\boldsymbol{x}$ and the demands $\boldsymbol{d}=(\tilde{\mathbf{d}})$ are observable at time $k$, and the pair $(\boldsymbol{A}, \boldsymbol{B})$ is controllable.

According to Assumption 1, the demand at time $k$ can be measured. But, the demand throughout the prediction horizon $H_{p}$ needs to be forecasted. Thus, we introduce the following assumption.

Assumption 2. The realization of actual demands $\tilde{\mathbf{d}}(k+i)$ at each future time instant $k+i$ may be decomposed as a summation of expected and uncertain additive demands

$$
\tilde{\mathbf{d}}(k+i)=\mathbf{d}(i \mid k)+\Delta \mathbf{d}(i \mid k) \quad i=1,2, \ldots, H_{p}
$$

where $\mathbf{d}(i \mid k)$ is the expected demand for the prediction horizon step $i$ at sampling time instant $k$, and $\Delta \mathbf{d}(i \mid k)$ is the error of this prediction.

The error $\Delta \mathbf{d}(i \mid k)$ is unknown but considered to be bounded

$$
\Delta \mathbf{d}(i \mid k) \in[-\Delta \theta, \Delta \theta] \quad i=1,2, \ldots, H_{p}
$$

where $\Delta \boldsymbol{\theta}$ are the bounds.

To propagate the effect of the uncertainty along the prediction horizon these bounds will be represented in a zonotopic form

$$
\Delta \mathbf{d}(i \mid k) \in \mathbf{0} \oplus \mathbf{H}_{d} \mathbf{z}_{d} \quad i=1,2, \ldots, H_{p}
$$

where: 0 is a column vector of $n_{d}$ zeros considered as the center of the zonotope, $\oplus$ denotes the Minkowski sum, $\mathbf{H}_{d}$ is a diagonal matrix defined as $\operatorname{diag}\left(\Delta \theta_{1}, \ldots, \Delta \theta_{n d}\right)$ and $\mathbf{z}_{d} \in \mathbb{B}^{n d}$ with $\mathbb{B}=[-1,1]$.

\section{B. Control objectives}

The main goal of the economic MPC control strategy is to minimize the use of the diesel generator and to encourage the use of renewable energy sources (PV, wind and hydroelectric generators). To achieve this end, we have designed the objective function in such a way that it includes the terms of renewable energy sources as well.
Hence, the objective function of the MPC controller consists of the following terms:

\section{B. 1 Economic cost:}

The total economic cost is given by:

$$
f_{E}(k)=\left(\boldsymbol{\alpha}_{1}+\boldsymbol{\alpha}_{2}(k)\right)^{T} \mathbf{u}(k) \Delta t
$$

where:

$\mathbf{u}(k)$ is a vector of control actions at time $k ; \Delta t$ is the sampling time in seconds; $\boldsymbol{\alpha}_{1}$ is a known vector related to economic costs of maintenance of generators and their accessories; $\boldsymbol{\alpha}_{2}(k)$ is a known time-varying vector associated to the economic cost of power flows related to transmission and distribution.

\section{B.2 Safety storage levels}

The safety objectives penalize the amount of power that goes below or above a pre-specified security threshold. They are defined as:

- Upper safety level:

$$
f_{s}^{+}(k)=\boldsymbol{\varepsilon}^{+}(k)^{T} \boldsymbol{\varepsilon}^{+}(k)
$$

- Lower safety level:

$$
f_{s}^{-}(k)=\boldsymbol{\varepsilon}^{-}(k)^{T} \boldsymbol{\varepsilon}^{-}(k)
$$

where $\boldsymbol{\varepsilon}^{-}(k)$ and $\boldsymbol{\varepsilon}^{+}(k)$ are the lower and upper safety limit violations.

\section{B.3 Smoothness of the control actions}

This objective is used to avoid peaks of power during the charging/discharging process of the storage elements that could damage them

$$
\mathrm{f}_{\Delta \mathrm{u}}(k)=\Delta \mathbf{u}(k)^{T} \Delta \mathbf{u}(k)
$$

where $\Delta \mathbf{u}(k)$ is the control signal rate of change, defined as $\Delta \mathbf{u}(k)=\mathbf{u}(k)-\mathbf{u}(k-1)$

\section{Formulation of the nominal MPC controller}

Taking into account the model of the smart micro-grid (1) and the control objective including (5)-(8), we can formulate the overall objective function as follows

$$
\begin{aligned}
& J_{E M P C}(i \mid k)=\left(\lambda_{1}\left(\boldsymbol{\alpha}_{1}+\boldsymbol{\alpha}_{2}(k)\right)^{T} \mathbf{u}(i \mid k)\right. \\
& +\lambda_{2}^{+} \boldsymbol{\varepsilon}^{+}(i \mid k)^{T} \boldsymbol{\varepsilon}^{+}(i \mid k)+\lambda_{2}^{-} \boldsymbol{\varepsilon}^{-}(i \mid k)^{T} \boldsymbol{\varepsilon}^{-}(i \mid k) \\
& \left.+\lambda_{3}[\mathbf{u}(i \mid k)-\mathbf{u}(i-1 \mid k)]^{T}[\mathbf{u}(i \mid k)-\mathbf{u}(i-1 \mid k)]\right)
\end{aligned}
$$

According to the geographical location of the renewable energy sources, we can construct some profiles of their power generations, and thereby it is possible to define some constraints (i.e. bounds) on their power generation. 
Based on those profiles along with energy storage elements, the dispatchability of renewable energies can be reasonably achieved.

We formulate the nominal MPC controller of the optimization problem as follows:

$$
\begin{aligned}
& \min _{\mathbf{u}\left(i \mid k \varepsilon^{+}(i k) \varepsilon^{-}(i \mid k)\right.} \sum_{i=0}^{H_{p}-1} J_{E M P C}(i \mid k) \\
& \text { s.t. } \\
& \mathbf{x}(i+1 \mid k)=\mathbf{A x}(i \mid k)+\mathbf{B u}(i \mid k)+\mathbf{B}_{d} \mathbf{d}(i \mid k) \\
& \mathbf{E}_{u} \mathbf{u}(i \mid k)+\mathbf{E}_{d} \mathbf{d}(i \mid k)=0 \\
& \mathbf{u}^{\min }(i \mid k) \leq \mathbf{u}(i \mid k) \leq \mathbf{u}^{\max }(i \mid k) \\
& \mathbf{x}^{\min } \leq \mathbf{x}(i+1 \mid k) \leq \mathbf{x}^{\max } \\
& \delta^{\min }(i \mid k)-\varepsilon^{-}(i \mid k) \leq \mathbf{x}(i \mid k) \leq \delta^{\max }(i \mid k)+\boldsymbol{\varepsilon}^{+}(i \mid k) \\
& \boldsymbol{\varepsilon}^{+}(i \mid k) \geq 0 \\
& \boldsymbol{\varepsilon}^{-}(i \mid k) \geq 0 \\
& \delta^{\min }(i \mid k) \geq 0 \\
& \delta^{\max }(i \mid k) \geq 0
\end{aligned}
$$

where: $\mathbf{u}^{\min }(k)$ and $\mathbf{u}^{\max }(k)$ are the lower and upper bounds of the control inputs; $\mathbf{x}^{\min }(k)$ and $\mathbf{x}^{\max }(k)$ are the lower and upper limits of the storage elements; $\lambda_{1}, \lambda_{2}^{+}, \lambda_{2}^{-}, \lambda_{3}$ are weighting coefficients for prioritizing the objectives.

Finally, in case of demands presenting repetitive periodic patterns, the prediction horizon $H_{p}$ is taken equal to the period, and we include the following constraint to guarantee effective usage of the storage elements.

$$
\mathbf{x}\left(H_{p} \mid k\right)=\mathbf{x}(0 \mid k)
$$

Noticeably, the MPC controller (10) is not robust against the demand uncertainty forecast defined in (2). Thus, in the next section, this controller will be robustified by taking into account the uncertainty bounds (3).

\section{ROBUSTYFING THE MPC CONTROLLER}

The proposed approach considers the effect of uncertainty by tightening the system constraints along the prediction horizon. The system control inputs are divided into dependent and independent variables. For instance, energy produced by the energy suppliers e.g. solar panels can be simultaneously used to satisfy load demands and charge the batteries. The energy suppliers are the independent variables while the consumers are the dependent variables. In this way, some particular dependent control variables are selected and bounded by zonotope to compensate for any discrepancy between predicted and actual demands, while the uncertainty propagates forward along the prediction horizon. Additionally, corresponding uncertain state estimation due to the uncertain demand has also been determined and bounded by a zonotope at each time instant along the prediction horizon. The bounds of the tightened constraints are computed offline.

\section{A. Decomposition of the control variables}

Eq. (1b) can be used to split the control input variables into independent and dependent variables. The aim is to develop an affine parameterization of the control input variables in terms of demand variables In fact, the affine dependence method has already been discussed in [1],[4],[8], [11].

Assumption 4. There are more variables than algebraic equations, i.e., $n_{q}<n_{u}$. The matrix $\mathbf{E}_{\mathrm{u}}$ in (1b) has maximal rank, i.e. $\operatorname{rank} \mathbf{E}_{\mathrm{u}}=n_{q}$.

We suppose that, control inputs can be partitioned into independent and dependent variables (for instance by applying LU-decomposition or simply heuristic approaches).

$$
\mathbf{u}(k)=\mathbf{P}_{1} \mathbf{u}_{\text {indep }}(k)+\mathbf{P}_{2} \mathbf{u}_{\text {dep }}(k)
$$

where $\mathbf{P}_{1} \in \mathfrak{R}^{n_{u} \times n_{\text {indep }}}$ and $\mathbf{P}_{2} \in \mathfrak{R}^{n_{u} \times n_{\text {dep }}}$ are suitable permutation matrices which define $\mathbf{u}$ from independent variables $\mathbf{u}_{\text {indep }}$ and dependent variables $\mathbf{u}_{\text {dep }}$.

Then, we can write

$$
\mathbf{E}_{u} \mathbf{u}(k)=\mathbf{E}_{\text {indep }} \mathbf{u}_{\text {indep }}(k)+\mathbf{E}_{\text {dep }} \mathbf{u}_{\text {dep }}(k)
$$

where $\mathbf{E}_{\text {indep }}=\mathbf{E}_{u} \mathbf{P}_{1}$ and $\mathbf{E}_{\text {dep }}=\mathbf{E}_{u} \mathbf{P}_{2}$

Assuming, dependent input vector will compensate future deviations of demand predictions defined in Eq (2), we decompose the actual predicted control input into two components:

$$
\tilde{\mathbf{u}}_{d e p}(i \mid k)=\mathbf{u}_{d e p}(i \mid k)+\Delta \mathbf{u}_{d e p}(i \mid k) \quad i=1,2, \ldots, H_{p}-1
$$

where $\mathbf{u}_{\text {dep }}(i \mid k)$ is the nominal prediction that is computed by nominal MPC problem (10) and $\Delta \mathbf{u}_{d e p}(i \mid k)$ is the component of dependent inputs that would compensate future unexpected additive demand, i.e. $\Delta \mathbf{d}(i \mid k)$ in Eq. (2).

On the other hand, we suppose that, the independent component will be given directly by MPC optimization problem (10) i.e.

$$
\tilde{\mathbf{u}}_{\text {indep }}(i \mid k)=\mathbf{u}_{\text {indep }}(i \mid k) \quad i=1,2, \ldots, H_{p}-1
$$

Substituting the actual demands (2) and the actual predicted inputs (14) into (13), we obtain

$$
\begin{aligned}
& \mathbf{E}_{\text {indep }} \mathbf{u}_{\text {indep }}(i \mid k)+\mathbf{E}_{\text {dep }}\left(\mathbf{u}_{\text {dep }}(i \mid k)+\Delta \mathbf{u}_{\text {dep }}(i \mid k)\right)= \\
& -\mathbf{E}_{\mathrm{d}}(\mathbf{d}(i \mid k)+\Delta \mathbf{d}(i \mid k))
\end{aligned}
$$

Furthermore, (16) can be split into deterministic and stochastic (uncertain) parts. The deterministic part that is considered in the MPC optimization problem (10) is given as: 


$$
\mathbf{E}_{\text {indep }} \mathbf{u}_{\text {indep }}(i \mid k)+\mathbf{E}_{\text {dep }} \mathbf{u}_{\text {dep }}(i \mid k)=-\mathbf{E}_{\mathrm{d}} \mathbf{d}(i \mid k)
$$

while the uncertain part is given by:

$$
\mathbf{E}_{d e p} \Delta \mathbf{u}_{d e p}(i \mid k)=-\mathbf{E}_{\mathrm{d}} \Delta \mathbf{d}(i \mid k)
$$

Assuming matrix $\mathbf{E}_{d e p}$ to be invertible, we can write

$$
\Delta \mathbf{u}_{d e p}(i \mid k)=-\mathbf{E}_{d e p}^{-1} \mathbf{E}_{\mathrm{d}} \Delta \mathbf{d}(i \mid k)
$$

Considering the zonotopic demand bounds (4), the dependent input vector can also be bounded with a zonotope as follows:

$\Delta \mathbf{u}_{d e p}(i \mid k) \in \mathbf{0} \oplus \overline{\mathbf{H}}_{d} \mathbf{z}_{d}$

with $\overline{\mathbf{H}}_{d}=-\mathbf{E}_{d e p}^{-1} \mathbf{E}_{\mathrm{d}} \mathbf{H}_{d}$

\section{B. Decomposition of the state variables}

Since the input controls can be decomposed into two components, we expect the states to be also decomposable into two parts.

The predicted evolution of the actual state can be expressed as

$$
\tilde{\mathbf{x}}(i+1 \mid k)=\mathbf{A} \tilde{\mathbf{x}}(i \mid k)+\mathbf{B} \tilde{\mathbf{u}}(i \mid k)+\mathbf{B}_{\mathrm{d}} \tilde{\mathbf{d}}(k+i) \quad i=1,2, \ldots
$$

Considering demand uncertainty and inputs decomposition leads to

$$
\begin{gathered}
\tilde{\mathbf{x}}(i+1 \mid k)=\mathbf{A} \tilde{\mathbf{x}}(i \mid k)+\mathbf{B}_{\text {ind }} \mathbf{u}_{\text {ind }}(i \mid k)+\mathbf{B}_{\text {dep }}\left(\mathbf{u}_{\text {dep }}(i \mid k)+\Delta \mathbf{u}_{\text {dep }}(i \mid k)\right) \\
+\mathbf{B}_{\mathrm{d}}(\mathbf{d}(i \mid k)+\Delta \mathbf{d}(i \mid k))
\end{gathered}
$$

where $\mathbf{B}_{\text {ind }}=\mathbf{B} \mathbf{P}_{1}$ and $\mathbf{B}_{\text {dep }}=\mathbf{B P}_{2}$.

Expanding (22) and applying demand error compensation, we obtain:

$$
\begin{aligned}
& \tilde{\mathbf{x}}(i+1 \mid k)=\mathbf{A} \tilde{\mathbf{x}}(i \mid k)+\mathbf{B}_{\text {ind }} \mathbf{u}_{\text {ind }}(i \mid k)+\mathbf{B}_{\text {dep }} \mathbf{u}_{d e p}(i \mid k)+\mathbf{B}_{\mathrm{d}} \mathbf{d}(i \mid k) \\
& +\left(-\mathbf{B}_{\text {dep }} \mathbf{E}_{\text {dep }}^{-1} \mathbf{E}_{\mathrm{d}}+\mathbf{B}_{\mathrm{d}}\right) \Delta \mathbf{d}(i \mid k)
\end{aligned}
$$

Now, the future state can be decomposed as

$$
\tilde{\mathbf{x}}(i+1 \mid k)=\mathbf{x}(i+1 \mid k)+\Delta \mathbf{x}(i+1 \mid k)
$$

where $\mathbf{x}(i+1 \mid k)$ is the deterministic estimation part considered in MPC optimization problem, and $\Delta \mathbf{x}(i+1 \mid k)$ is the uncertain part due to the demand error estimation.
Considering that $\tilde{\mathbf{x}}(0 \mid k)=\tilde{\mathbf{x}}(k)$ and $\tilde{\mathbf{d}}(k)$ are known at time $k$, and $\tilde{\mathbf{u}}_{\text {ind }}(k), \tilde{\mathbf{u}}_{\text {dep }}(k)$ are computed by the MPC optimization problem at time $k$, we can write:

$$
\tilde{\mathbf{x}}(k+1)=\mathbf{A} \tilde{\mathbf{x}}(k)+\mathbf{B}_{i n d} \tilde{\mathbf{u}}_{i n d}(k)+\mathbf{B}_{d e p} \tilde{\mathbf{u}}_{d e p}(k)+\mathbf{B}_{\mathrm{d}} \tilde{\mathbf{d}}(k)
$$

And the deterministic state estimation part can be given by

$\mathbf{x}(i+1 \mid k)=\mathbf{A x}(i \mid k)+\mathbf{B} \mathbf{u}(i \mid k)+\mathbf{B}_{d} \mathbf{d}(i \mid k) \quad i=1,2, \ldots$

with

$\mathbf{x}(1 \mid k)=\tilde{\mathbf{x}}(k+1)$ computed by means of $(25)$ and

$\mathbf{u}(i \mid k)=\mathbf{P}_{1} \mathbf{u}_{\text {indep }}(i \mid k)+\mathbf{P}_{2} \mathbf{u}_{\text {dep }}(i \mid k)$

Iteratively computing the state of the system at each time instant $k$, we can express the uncertain state estimation part $\Delta \mathbf{x}(i+1 \mid k)$ as an accumulative function of the unexpected additive demands

$\Delta \mathbf{x}(i+1 \mid k)=\sum_{j=1}^{i}\left(\mathbf{A}^{i-j}\left(-\mathbf{B}_{d e p} \mathbf{E}_{d e p}^{-1} \mathbf{E}_{\mathrm{d}}+\mathbf{B}_{\mathrm{d}}\right) \Delta \mathbf{d}(i \mid k+j)\right) \quad i=1,2, \ldots$

with $\Delta \mathbf{x}(1 \mid k)=0$.

Now, considering demand bounds (2), the uncertain state estimation part can also be bounded with the following zonotope:

$$
\Delta \mathbf{x}(i+1 \mid k) \in \mathbf{0} \oplus \overline{\mathbf{H}}_{x}(i) \overline{\mathbf{z}}_{d_{i}} \quad i=1,2,3, \ldots
$$

with $\overline{\mathbf{z}}_{d_{i}}{ }^{t}=\left[\begin{array}{lll}\mathbf{z}_{d_{1}}{ }^{t} & \cdots & \mathbf{z}_{d_{i}}{ }^{t}\end{array}\right]$ where $\mathbf{z}_{d_{j}} \in \mathbb{B}^{\text {nd }} j=1, \ldots, i$ and

$$
\overline{\mathbf{H}}_{x}(i)=\left[\begin{array}{llll}
\overline{\mathbf{H}}_{d X} & \mathbf{A} \overline{\mathbf{H}}_{d X} & \cdots & \mathbf{A}^{i-1} \overline{\mathbf{H}}_{d X}
\end{array}\right]
$$

where $\overline{\mathbf{H}}_{d X}=\left(-\mathbf{B}_{d e p} \mathbf{E}_{d e p}^{-1} \mathbf{E}_{\mathrm{d}}+\mathbf{B}_{\mathrm{d}}\right) \mathbf{H}_{\mathrm{d}}$.

\section{FORMULATION OF THE ROBUST MPC}

\section{A. Tightening the MPC variable bounds}

Once the effect of demand uncertainty are propagated to the control inputs and states, input and state constraints in the MPC problem (10) need to be adjusted using interval arithmetic, such that actual predicted inputs and states do not violate the defined input and state bounds i.e

$$
\begin{aligned}
& \mathbf{u}^{\text {min }}(i \mid k) \leq \tilde{\mathbf{u}}(i \mid k) \leq \mathbf{u}^{\max }(i \mid k) \\
& \mathbf{x}^{\text {min }} \leq \tilde{\mathbf{x}}(i+1 \mid k) \leq \mathbf{x}^{\max }
\end{aligned}
$$

Then, we can reformulate the MPC optimization problem (10) in a robust way as follows: 


$$
\begin{aligned}
& \min _{\left.\mathbf{u}(i \mid k) \varepsilon^{+}(i \mid k) \varepsilon^{-(}(i k)\right)} \sum_{i=0}^{H_{p}-1} J_{E M P C}(i \mid k) \\
& \text { s.t. } \\
& \mathbf{x}(i+1 \mid k)=\mathbf{A x}(i \mid k)+\mathbf{B u}(i \mid k)+\mathbf{B}_{d} \mathbf{d}(i \mid k) \\
& \mathbf{E}_{u} \mathbf{u}(i \mid k)+\mathbf{E}_{d} \mathbf{d}(i \mid k)=0 \\
& u_{\text {indep }, j}^{\min }(i \mid k) \leq u_{\text {indep }, j}(i \mid k) \leq u_{\text {indep }, j}^{\max }(i \mid k) j=1, \ldots, n_{\text {indep }} \\
& u_{\text {dep }, j}^{\min }(i \mid k)+\Delta_{\text {dep }, j} \leq u_{\text {dep }, j}(i \mid k) \leq u_{\text {dep }, j}^{\max }(i \mid k)-\Delta_{\text {dep }, j} \\
& x_{j}^{\min }+\Delta_{x, j}(i) \leq x_{j}(i \mid k) \leq x_{j}^{\max }-\Delta_{x, j}(i) \\
& \delta^{\min }(i \mid k)-\varepsilon^{-}(i \mid k) \leq \mathbf{x}(i \mid k) \leq \delta^{\max }(i \mid k)+n_{\text {dep }} \\
& \varepsilon^{+}(i \mid k) \geq 0 \\
& \varepsilon^{-}(i \mid k) \geq 0 \\
& \delta^{\min }(i \mid k) \geq 0 \\
& \delta^{\max }(i \mid k) \geq 0 \\
& \mathbf{x}\left(H_{p} \mid k\right)=\mathbf{x}(0 \mid k)
\end{aligned}
$$

with $\Delta_{d e p, j}$ and $\Delta_{x, j} \quad$ computed using interval arithmetic[13],[14],[15].

\section{APPLICATION}

\section{A. Description}

In this section, the applicability of the proposed method will be assessed using a smart micro-grid comprising two clusters linked through two DC-buses that are connected through a switch. The first cluster is primarily used to satisfy residential demands, and it comprises some photovoltaic panels, a diesel generator, and a lead-acid battery. The second cluster consisting of wind and hydroelectric generators, and one virtual sink (external grid connection) is used to satisfy industrial and other DC-load demands. A grid connection is a sink when it is buying energy, and a source when selling energy. The block diagram of the smart micro-grid is shown in Figure 1.

\section{B. Control-oriented Model}

All the components are considered as manipulated inputs (excluding sinks). The states of the smart grid are defined to be the state of charge of the storage elements.

\section{State variables:}

$x_{b}$ and $x_{h}$ are the state of charge (SOC) of the batteries (leadacid and hydrogen respectively): $\mathbf{x}(k) \triangleq\left[x_{b}(k), x_{h}(k)\right]^{\mathrm{T}}$

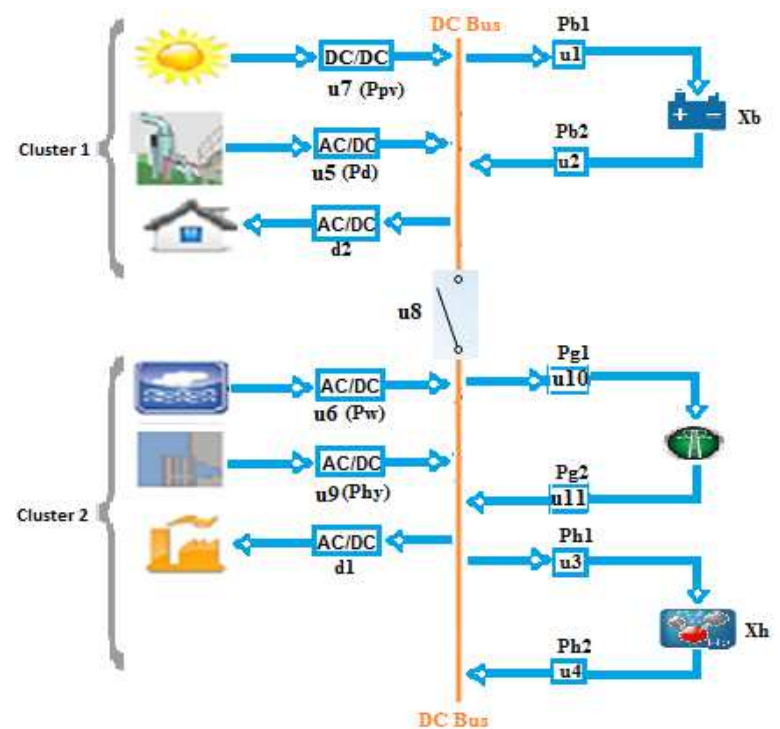

Figure 1. Block diagram of the smart micro-grid

Control input variables:

$\mathbf{u}(k) \triangleq\left[P_{b 1}(k), P_{b 2}(k), P_{h 1}(k), P_{h 2}(k), P_{d}(k), P_{w}(k), P_{p v}(k), P f(k)\right.$, $\left.P_{h y}(k), P_{g l}(k), P_{g 2}(k),\right]^{\mathrm{T}}$

where $P_{b 1}$ and $P_{b 2}$ are the charged power and discharged power of the lead-acid battery; $P_{h 1}$ and $P_{h 2}$ are the charged and discharged power of the hydrogen battery; $P_{g 1}$ and $P_{g 2}$ are the exported and imported power into/from the external grid; $P_{d}$, $P_{h y}, P_{p v}$, and $P_{w}$ stand for the power supplied to the DC Buses by the diesel, hydroelectric, wind, and photovoltaic generators respectively. $P f$ is the power flow from Bus 2 to Bus 1 .

\section{Disturbance variables:}

$d_{1}$ is the industrial load, $d_{2}$ is the residential load. The disturbance vector $\mathbf{d}$ consists of the two loads:

$\mathbf{d}(k) \triangleq\left[d_{1}(k), d_{2}(k)\right]^{\mathrm{T}}$

The matrices and vectors that define the system and its constraints are given as follows:

$$
\begin{aligned}
\mathbf{A} & =\left(\begin{array}{ll}
1 & 0 \\
0 & 1
\end{array}\right) \\
\mathbf{B} & =\left(\begin{array}{ccccccccccc}
\eta_{\mathrm{bc}} & -\eta_{\mathrm{bd}} & 0 & 0 & 0 & 0 & 0 & 0 & 0 & 0 & 0 \\
0 & 0 & \eta_{\mathrm{hc}} & -\eta_{\mathrm{hd}} & 0 & 0 & 0 & 0 & 0 & 0 & 0
\end{array}\right) \\
\mathbf{B}_{\mathrm{d}} & =\left(\begin{array}{cc}
0 & 0 \\
0 & 0
\end{array}\right)
\end{aligned}
$$

where:

$\eta_{h c}$ and $\eta_{h d}$ are the charging efficiency and discharging efficiency of the hydrogen battery respectively; and $\eta_{b c}$ and $\eta_{b d}$ are the charging efficiency and discharging efficiency of the lead-acid battery respectively.

$\mathbf{x}^{\min }=\left[\begin{array}{ll}0 & 0\end{array}\right]^{\mathrm{T}}, \mathbf{x}^{\max }=\left[\begin{array}{lll}100 & 100\end{array}\right]^{\mathrm{T}}$

$\mathbf{u}^{\min }(k)=\left[\begin{array}{lllllllllll}0 & 0 & 0 & 0 & 0 & 0 & 0 & 0 & 0 & 0 & 0\end{array}\right]^{\mathrm{T}}$,

$\mathbf{u}^{\max }(k)=\left[\begin{array}{lllll}2.2 & 10.2 & 2.2 & 10.2 & 7.7500 P p_{w}(k) P p_{p v}(k) \\ 2.2 & 8 & 3 & 3\end{array}\right]^{T}$

where $P p_{w}(k) \leq 7.75 \mathrm{Kw}$ and $P p_{p v}(k) \leq 6.75 \mathrm{~kW}$ are the energy 
generation profiles of the wind and photovoltaic generators respectively. The static equations are defined through

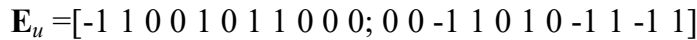

$$
\begin{aligned}
& \mathbf{E}_{d}=[-10 ; 0-1]
\end{aligned}
$$

The additive uncertain demand $\Delta \mathbf{d}(i \mid k)$ is considered to be bounded by a box defined in zonotopic form (4) as

$$
\mathbf{H}_{d}=\left(\begin{array}{cc}
\Delta \theta_{1} & 0 \\
0 & \Delta \theta_{2}
\end{array}\right) \text { and } \Delta \theta_{1}=\Delta \theta_{2}=0.7
$$

On the other hand, the charging batteries' inputs $u_{1}(k)$ and $u_{3}(k)$ have been chosen as dependent variables.

Initial values of the subsystems, as well as the state of charge of the batteries are set to zero. The simulations were made for 96 hours (4 days). The diesel and hydroelectric generators delivered between 1-1.3 kWh in summer during the first six hours of the day, and in winter in the afternoon for six hours. The batteries delivered between 1-2 $\mathrm{kWh}$ during the first two hours of the day. $1 \mathrm{kWh}$ was bought from the external grid during the second hour of the day.

\begin{tabular}{|ll|lll|}
\hline \multicolumn{2}{|l|}{ Model parameters } & \multicolumn{1}{|c|}{ Energy prices (e.u) } & \\
$\eta \mathrm{bc}$ & 0.95 & Lead-acid battery charging & $:$ & 0.02 \\
$\eta \mathrm{bd}$ & 1 & Lead-acid battery discharging: & 0.02 \\
$\eta \mathrm{hc}$ & 0.85 & Hydrogen battery charging & $:$ & 0.02 \\
$\eta \mathrm{hd}$ & 1.0 & Hydrogen battery discharging: & 0.02 \\
$\Delta$ & {$[3535]^{\mathrm{T}}$} & Power flow between node: & 0.02 \\
$\mathrm{H}_{\mathrm{p}}$ & 24 & External grid selling: & 2.0 \\
$\lambda 1$ & 2500 & External grid buying: & 2.0 \\
$\lambda 2$ & 12 & Diesel: & 4.3 \\
$\lambda 3$ & 0.1 & Hydroelectric: & 1.8 \\
& & Wind: & 1.7 \\
\multicolumn{7}{r}{} \\
\hline
\end{tabular}

Figure 2 and 3 show some expected profiles of PV and wind generator, as well as load demands. The additive uncertain demand is represented with the shadowed green area in Figure 3 .

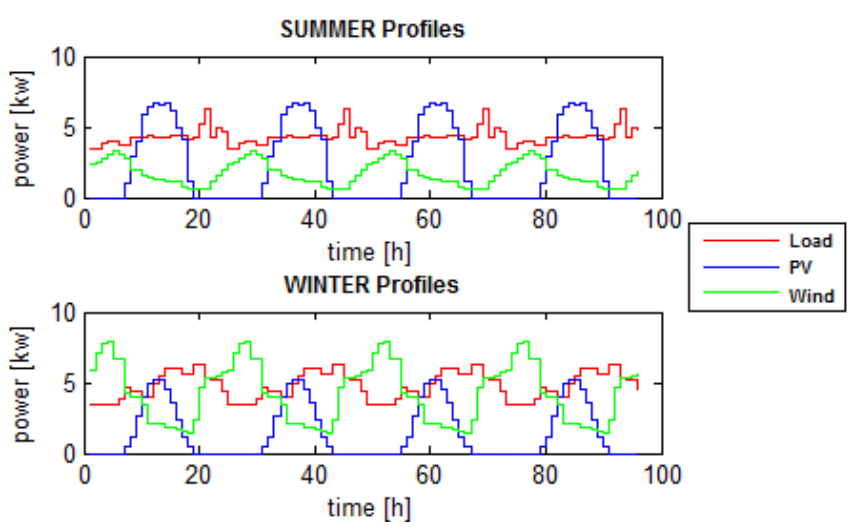

Figure 2. Forecasted profiles of PV and wind generators, and load demands
The profile of a generator represents the maximum power that can be ideally produced by the generator.
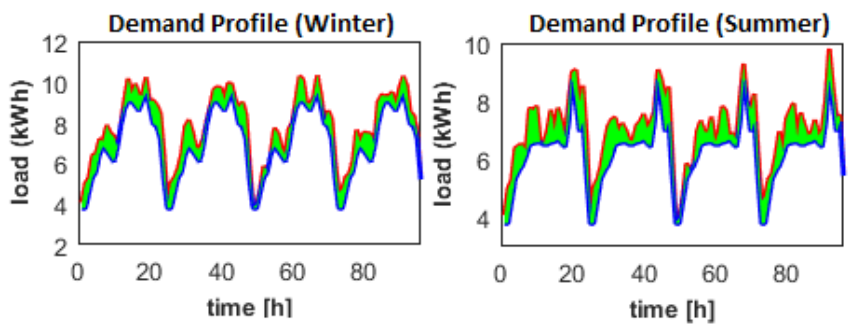

Figure 3. Load demands' profiles

\section{Results}

The proposed MPC strategy has been implemented using YALMIP (CPLEX solver) within the Matlab environment.

Table 2 shows an economic comparison between the nominal system (i.e. neglecting uncertain demands) and the proposed robust method. As expected, the demand uncertainty increases the overall energy production costs, but the proposed method provides robustness against unexpected changes in the demands.
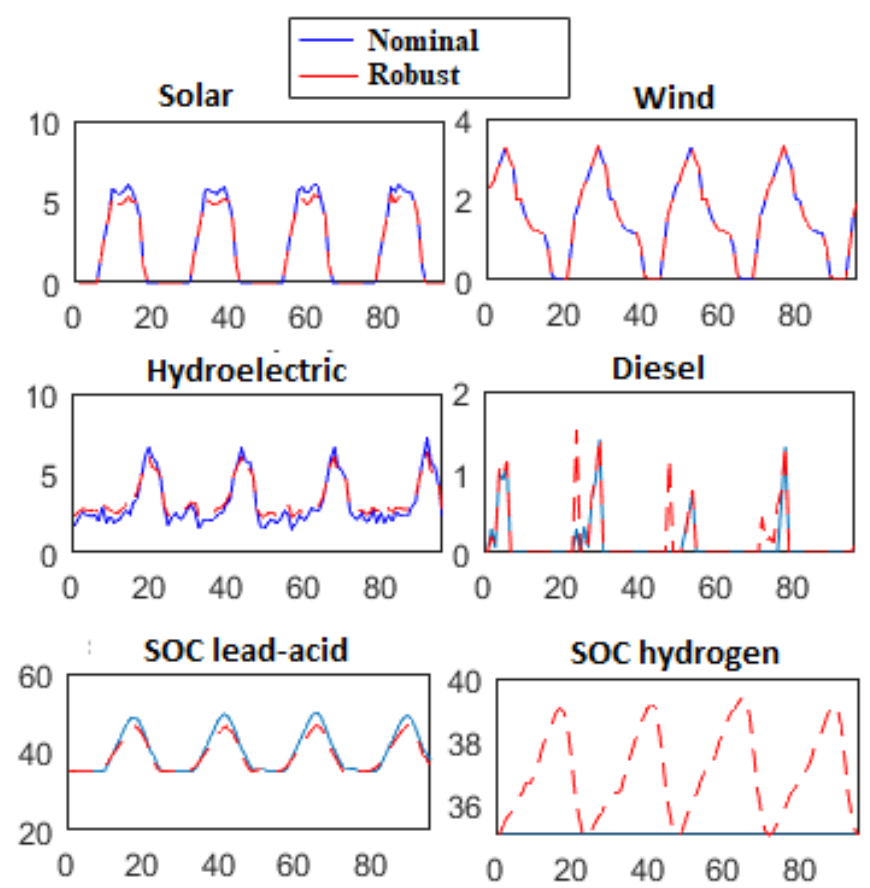

Figure 4. Sample summer plots of energy production 

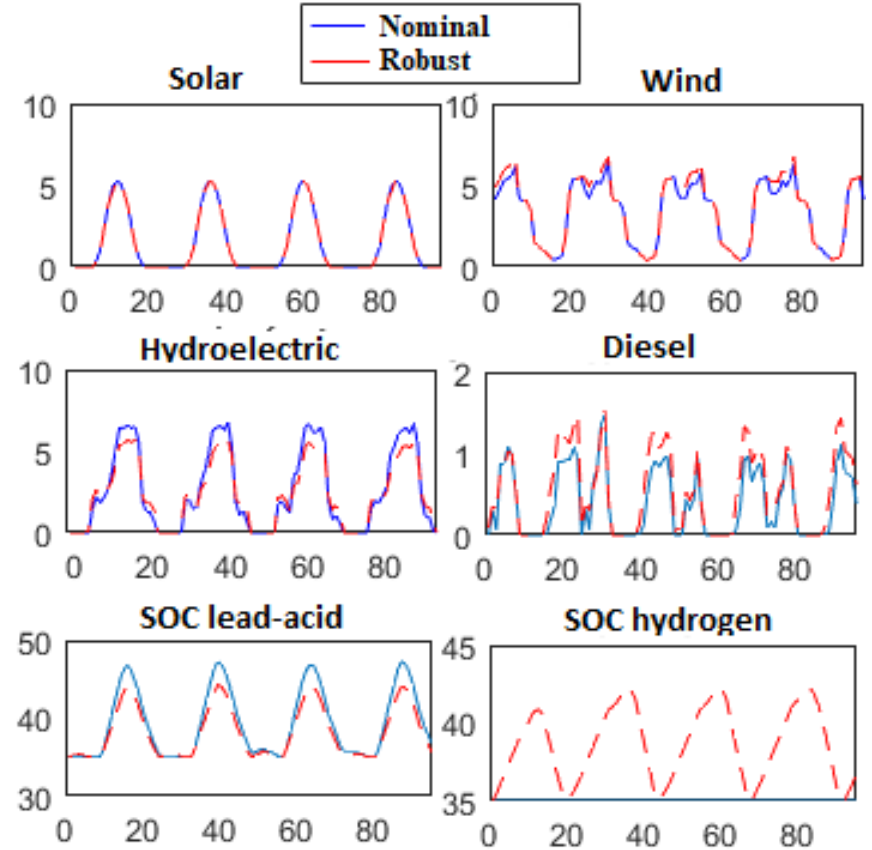

Figure 5. Sample Winter plots of energy production

\begin{tabular}{|c|c|c|}
\hline & $\begin{array}{c}\text { Nominal } \\
\text { EMPC }\end{array}$ & $\begin{array}{c}\text { Robust } \\
\text { EMPC }\end{array}$ \\
\hline Summer & 568.02 & 587.72 \\
\hline Winter & 676.66 & 706.10 \\
\hline
\end{tabular}

Table 2. Daily economic costs.

\section{CONCLUSIONS}

In this work, a robust economic MPC strategy for optimally controlling an smart micro-grid considering uncertainty in the demand forecasts that is assumed unknown but bounded. Uncertain load demands are unknown but bounded by zonotopes. The proposed robust economic model predictive control strategy is based on tightening in real-time the constraints of the control inputs. Control inputs have been decomposed into independent and dependent components, and then the dependent component has further been divided into two parts, whereby one of the parts is bounded by a zonotope and is used to compensate for any deviation of the actual demand from the forecasted one. Additionally, a corresponding uncertain state estimation of the uncertain demand has also been determined and bounded by a zonotope Finally, the results of a case study have shown that, the proposed robust EMPC method can be successfully used to solve the problem of energy dispatch considering demand uncertainty in electrical smart micro-grids. The next steps for completing this work will be devoted to the development of an automatic method of successively selecting optimal control inputs for compensating demand deviations. Additionally, extending the developed method to include uncertainty of energy prices and renewable energies will also be carried out.

\section{ACKNOWLEDGMENT}

This work has been partially funded by the Spanish State Research Agency (AEI) and the European Regional Development Fund (ERFD) through the projects DEOCS (ref. MINECO DPI2016-76493) and SCAV (ref. MINECO DPI2017-88403-R). This work has also been partially funded by AGAUR of Generalitat de Catalunya through the Advanced Control Systems (SAC) group grant (2017 SGR 482). J. Blesa acknowledges the support from the Serra Húnter program.

\section{REFERENCES}

[1] Abate, A., and Ghaoui, Laurent El., (2004) Robust model predictive control through adjustable variables: an application to path planning 43rd IEEE Conference on Decision and Control, (Atlantis, Paradise Island, Bahamas)

[2] Bemporad, A., and Morari, M., (1999) Robust model predictive control: A survey hybrid systems Computation and Control, F.W. Vaandrag and J.H. van Schuppen, vol. 1569, pp. 31-45,1999, Lecture in Computer Science.

[3] Ben-Tal, A., and Nemirovski, A., (1998) A Robust convex optimization Mathematics of Operations Research, Vol. 23, No. 4, Nov. 1998, pp. 769-805.

[4] Ben-Tal, A., Goryashko, A., Guslitzer, E., Nemirovski, A., (2004) Adjustable solutions of uncertain linear programs Mathematical Programming, Vol. 99, 2004, pp. 351-376.

[5] Loefberg, J., (2003) Minimax approaches to robust model predictive control Ph.D. dissertation, Univ. of Linkoping.

[6] Löfberg, J. (2012) Automatic robust convex programming Optimization methods and software, 27(1):115-129, 2012.

[7] Montes De Oca, S., Puig,V., and Blesa, J. (2012). Robust fault detection based on adaptive threshold generation using interval LPV observers. International Journal of Adaptive Control and Signal Processing, 26(3), 258-283.

[8] Nassourou, M., Puig. V., and Blesa, J., (2016) Robust Optimization based Energy Dispatch in Smart Grids Considering Demand Uncertainty, 13th European Workshop on Advanced Control and Diagnosis, 2016, Lille, Vol 783 of Journal of Physics: Conference Series, pp. 012033, 2017

[9] Nassourou, M., Puig. V., and Blesa, J., (2017) Robust Optimization based Energy Dispatch in Smart Grids Considering Simultaneously Multiple Uncertainties: Load Demands and Energy Prices, 20th World Congress of the International Federation of Automatic Control, 9-14 July 2017, Toulouse, France

[10] Ocampo-Martinez, C., Puig, V., Cembrano, G., Creus, R., and Minoves, M. (2009) Improving water management efficiency by using optimization-based control strategies: The Barcelona case study Water Science \&Technology: Water supply, vol. 9, no. 5, pp. 565-575

[11] Prodan, I., Enrico, Z., (2013) Predictive control for reliable microgrid energy management under uncertainties 22nd European Safety and Reliability (ESREL 2013) (Amsterdam, Netherlands) <hal-00912003>

[12] Ben-Tal, A., El Ghaoui, L., and Nemirovski, A., (2009) Robust Optimization, ISBN 978-0-691-14368-2, Published by Princeton University Press, 41 William Street, Princeton, New Jersey

[13] Jaulin, L., Kieffer, M., Didrit, O., and Walter, E. 2001 Applied Interval Analysis: with Examples in Parameter and State Estimation, Robust Control and Robotics. Springer.

[14] Blesa, J., Puig, V., and Saludes, J. 2011. Identification for passive robust fault detection using zonotope-based set-membership approaches. International Journal of Adaptive Control and Signal Processing, 25(9), 788-812.

[15] Blesa, J., Puig, V., and Saludes, J. (2012b). Robust identification and fault diagnosis based on uncertain multiple input-multiple output linear parameter varying parity equations and zonotopes. Journal of Process Control, 22(10), $1890-1912$. 